\title{
Fault Diagnosis Method of HV Circuit Breaker Based on Wavelets Neural Network
}

\author{
Liu Mingliang ${ }^{1,2}$, Wang Keqi ${ }^{1, *}$, Sun Laijun ${ }^{2}$ and Zhang Jianfeng ${ }^{2}$ \\ ${ }^{1}$ College of Mechanical and Electrical Engineering, Northeast Forestry University, Harbin 150040, China \\ ${ }^{2}$ HLJ Province Key Lab of Senior-Education for Electronic Engineering (Heilongjiang University) Harbin 150080 \\ China
}

\begin{abstract}
Fault diagnosis of HV circuit break has been investigated extensively as an important device in the field of power system. In view of the shortcoming of the traditional neural network, such as the slow convergence rate and the local minimum easy to form, fault diagnosis method of $\mathrm{HV}$ circuit breaker is proposed to remedy the defects of traditional neural network based on wavelets neural. This method adopts wavelets function rather than the hidden nodes of traditional neural network, which is propitious to conducive to achieve a rapid convergence of online learning. This work firstly discussed the principles of fault diagnosis method in detail, and then compared diagnosis effect using wavelets function with that of the traditional neural network. The results show that the training speed and classification effect of wavelets neural network are superior obviously to those of traditional neural network. Wavelets neural network based on vibration signals is more suitable in application to the fault diagnosis of HV circuit breakers.
\end{abstract}

Keywords: Wavelets neural network, HV circuit breaker, fault diagnosis.

\section{INTRODUCTION}

As an important equipment of power system, HV circuit breaker shoulders important responsibilities in collocating power-grid structure, protecting grid devices and isolating faults. Its running status, especially the mechanical conditions, are greatly concerned and emphasized by engineers and technicians. Therefore, researches of fault diagnosis have been launched successively [1-3].

Many experts and scholars proposed kinds of effective detection methods in the past researches of fault diagnosis based on vibration signals. In 1996, Runde and other experts made use of windowing FFT and DTW to analyze vibration signals and estimate time migration of vibration signals under normal condition and test condition. In the end, they achieved good diagnosis through comparing estimated time migrations with reference data [4]. Hu Xiaoguang and his team adopted multi-layer wavelet-packet decomposition envelope peak value to extract signal singularity indexes. Then, they used those indexes as the characteristic parameters to reflect the status changes of circuit breaker [5]. Dennis and his team applied wavelet package to resolve 4 signals of one breaker action. They figured out sensitive nodes of circuit breaker under abnormal conditions. Those nodes formed a node rectangular coordinate graph, showing the state comparison of the 4 signal parts. Then, a back propagation neural network was applied to classify statuses [6]. In the end, they achieved precise detection. There is a common point in above researches: all the above methods extracted characteristic parameters from vibration signals; then expert knowledge or neural network was used to analyze the state of circuit breakers. However, there still are many problems in actual application. For example, expert knowledge is too rigid for fault judgment, and knowledge summaries are different among individuals. Besides, the classification of traditional neural network requires long-time trainings with a large amount of data samples, and it is easy to trap into local minimum and hard to converge.

Wavelet neural network is the combination of wavelet theory and neural network. It has a fast convergence rate with simple learning algorithm. Therefore, it is applied in various aspects such as adaptive area [7], signal filtering [8, 9], pattern identification $[10,11]$, fault diagnosis and detection $[12,13]$. When translation and scaling of wavelet basis function is determined in advance, weight coefficient becomes the adjustable parameter of wavelet neural network. Wavelet network and its weight are in linear relationship, soit is conducive to avoid the defects of normal BP neural network. Therefore, wavelet neural network is very necessary for mechanical fault classification.

Based on wavelet neural network as the core of this research, the work proposed a new method to diagnose the fault of HV circuit breaker. Firstly of all, wavelet packet characteristic entropy (WP-CE) method was used to process signals coming from breaker actions and to extract parameters. Then, wavelet neural network model was set up to analyze and classify feature signals. Meanwhile, wavelet neural 


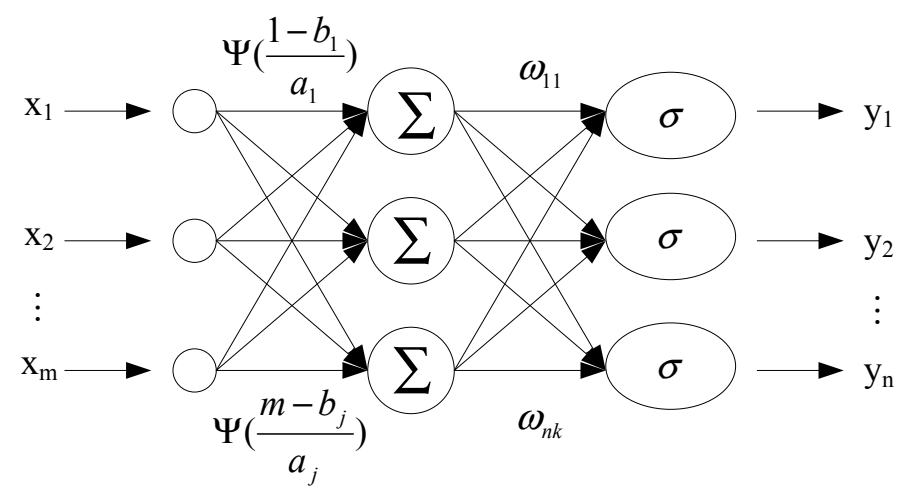

Fig. (1). The structure of wavelet neural network.

network and traditional BP neural network were compared in the work. According to experiments results, wavelet neural network is superior to traditional BP neural network in both classification effects and training speed.It replaces nonlinear activation function of normal neural network based on nonlinear wavelet. Thus, it is especially suitable to diagnose mechanical fault of HV circuit breaker.

\section{BASIC PRINCIPLES OF WAVELET NEURAL NETWORK}

Wavelet analysis is a mathematical theory and method developed from the mid-Eighties of twentieth century. Its basic concept is similar to Fourier transform. Fourier transform has no resolution in time domain and frequency domain. Besides, it cannot conduct local analysis. However, wavelet analysis remedies these defects with time-localized "window function", which is of great theoretical and practical significance [14]. Neural network is a new information processing system established from imitating the structure and function of human brain neuron and thinking ways. It is of high self-organization, self-adaptation and flexibility. Meanwhile, wavelet neural network is a new intelligent nonlinear network. It combines the characteristics of wavelet transform (time-frequency localization and zooming function) and neural network (self-learning, self-adaptation, fault tolerance and generalization ability). Wavelet neural network does not apply wavelet analysis to obtain signal characteristics and learning characteristic vectors. Instead, it adopts wavelet function to replace hidden node function of normal neural network. Besides, corresponding weights and thresholds from input layer to hidden layer are replaced by the scaling and translation parameters of wavelet function. Thus, activation function is the determined by wavelet function basis; the connection between wavelet transform and neural network is established through affine transformation [15].

Wavelet neural network in signal classification and identification tries to figure out a set of suitable wavelets in wavelet feature space. Thus, the minimum cost function can be obtained through network learning and self-adaptive adjustment. Fig. (1) shows the network structure:
$y_{i}(t)=\sigma\left[\sum_{j=1}^{J} \omega_{i} \sum_{k=1}^{m} x_{k}(t) \Psi\left(\frac{k-b_{j}}{a_{j}}\right)\right]$

Where $i=1,2, \ldots, \mathrm{n}, x_{k}(t)$ refers to the $k$ th input variable, $y_{i}(t)$ the $i$ th output variable, $j$ the hidden-layer unit number. Wavelet function $\Psi\left(\frac{k-b_{j}}{a_{j}}\right)$ is the weight function from the $k$ th input layer to the $j$ th hidden layer; $\omega_{i j}$ the connection weight from the $i$ th hidden layer to the $j$ th output layer. $\sigma$ is Sigmoid function. According to the function, $\omega_{i j}, a_{j}$ and $b_{i}$ are parameters waiting for training, and they can be learned with input samples $x_{k}(t)$ and teacher samples $g_{i}(t)$ through energy function minimization. Given the total number of input sample is $p_{0}$, energy function can figure out:

$E=\frac{1}{2} \sum_{p=1}^{p_{0}} \sum_{i=1}^{n}\left|g_{i}^{p}(t)-y_{i}^{p}(t)\right|^{2}$

Given $n_{0}$ is iterations, momentum factor $\alpha$ is introduced in to accelerate convergence and prevent oscillation; then modification iterative formula of weight changes at each layer can be obtained:

$$
\begin{aligned}
& a_{1}\left(n_{0}+1\right)=a_{j}\left(n_{0}\right)-\eta \frac{\partial E}{\partial a_{j}}+\sigma \Delta a_{j}\left(n_{0}\right) \\
& b_{1}\left(n_{0}+1\right)=b_{j}\left(n_{0}\right)-\eta \frac{\partial E}{\partial b_{j}}+\sigma \Delta b_{j}\left(n_{0}\right) \\
& \omega_{11}\left(n_{0}+1\right)=\omega_{i j}\left(n_{0}\right)-\eta \frac{\partial E}{\partial \omega_{i j}}+\sigma \Delta \omega_{i j}\left(n_{0}\right)
\end{aligned}
$$

Where $\eta$ is step length. When $\alpha=0$, this algorithm is a normal gradient descent learning method.

Formula (1) shows the network equation: 


\section{EXTRACTION OF CHARACTERISTIC PARAME-} TER

\subsection{Wavelet Packet Node Envelope Extraction}

First of all, we made use of wavelet packet method to conduct 3-layer decomposition to vibration signals. Then, signal nodes were reconstructed respectively at the 8 nodes in the third layer. After that, Hilbert transform was adopted to extract each node signal envelope as follows:

$$
A(t)=\sqrt{x^{2}(t)+\hat{x}^{2}(t)}
$$

where $x(t)$ is original signal; $\hat{x}(t)$ the Hilbert transform of $x(t)$,

\subsection{Calculation of Term Entropy}

We used wavelet packet method (introduced in 3.1) to extract each 3rd-layer node envelope with normal stand signal. Then, we divided envelope signal into equal $\mathrm{N}$ parts according to equivalent energy rule. Following that, we extracted time separation point in each section to separate corresponding node envelope for testing signals [16]. Finally, energy $Q(i)$ was figured out for each section of testing signal-envelope. Take the $k$ th node envelope signal as an example:

$Q_{k}(i)=\int_{t_{0}}^{t_{1}}|f(x)|^{2} d x$

where $k=0,1, \ldots 7, k=0,1, \ldots 7, t_{0}$ and $t_{1}$ the time boundaries of the $i$ th section. Define

$$
\varepsilon_{k}(i)=\frac{Q_{k}(i)}{\sum_{i=1}^{N} Q_{k}(i)}
$$

According to basic entropy principles, wavelet packet term entropy is defined as:

$$
H_{k}=-\sum_{i=1}^{N} \varepsilon_{k}(i) \lg \varepsilon_{k}(i)
$$

Where $H_{k}$ is the $k$ th wavelet-packet term entropy of vibration signal $u(t)$ in the third layer.

Then, we respectively extracted testing signal wavelet packet and resolved 8 wavelet packet term entropies in the third layer. Thus, we got term entropy vector $T$ :

$$
T=\left[H_{0}, H_{1}, H_{2}, H_{3}, H_{4}, H_{5}, H_{6}, H_{7}\right]
$$

According to the method, characteristic parameters reflected the differences between testing signals and normal signals from two aspects: the happening time and characteristic frequency of vibration events. After multiple tests, the number of decomposed layer was determined according to the frequency component of vibration signals. Thus, this number should be in line with specific condition. If there are many decomposed layers, corresponding frequency section of each node will be relatively narrow, which is sensitive to vibration frequency changes. If there are few layers, it will be slow in response. This is not good for judging the status. The same is true to the numbers of decomposed sections. If there are many decomposed sections, the parameters will be sensitive to the migration of the event time. If there are few decomposed sections, it can hardly reflect the migration. At a result, it is not good for detecting status changes. Refer to relative references $[17,18]$ for detailed method to extract characteristic parameter.

\section{EXPERIMENT ANALYSIS}

\subsection{Signal Extraction and Treatment}

ZW32 vacuum breaker was used for testing in this work. Its vibration transducer was installed inside of each phasefoundation supporting shim. Under the condition of no load, we made use of breaker's insufficient lubrication to detect time-delay faults. Besides, we loosened A phase base crews to detect loosened-state faults. Then, we applied on-line data-monitoring collector to collect vibration signals of the foundation. The collector was installed inside of breaker control box. Finally, the data was transmitted to PC through RS485 bus for treatment in main control room. The breaker made 20 actions under fault state and 20 under normal state. Therefore, 60 sets of A-phase fault data were obtained. Fig. (2) shows A-phase original vibration signals. More specifically, normal-state signals, operation signals (fault I, 3 signals) under screw-loosened state and operation signals (fault II, 3 signals) under time-delayed state were respectively presented in Fig. (2) from the top to the bottom.

First of all, we conducted wavelet soft-threshold denoising processing for collected data. After multiple tests, we selected threshold 2.1 according to de-noising effects. Thus, reconstructed and de-noised vibration signals were obtained. Fig. (3) implies obvious effects of de-noising treatment. Then, with Daubechies10 wavelet as wavelet basis function, we conducted three-layer decomposition to de-noised data. And, we reconstructed signals based on node coefficients in the third layer. In the end, we applied Hilbert transform to respectively extract the envelopes of reconstructed signals for the 8 nodes.

Here, we processed standard signals under normal state first. As for wavelet-packet decomposed node-envelope signals in the third layer, we divided the time shaft of each node into 15 sections according to equal energy principles. Table 1 shows the section separation time of each node. According to Table $\mathbf{1}$, there is relatively small gap between breaker vibration events. The smallest gap is only $5 \mathrm{~ms}$. However, the section gap at other flat parts is relatively big. This separation method helps to detect the time migration of each vibration event within each frequency section. Thus, just a parameter can reflect the changes of vibration signals in both time domain and frequency domain. 
Table 1. Time points of each equal-energy segment (Unit: ms).

\begin{tabular}{|c|c|c|c|c|c|c|c|c|}
\hline & {$[3,0]$} & {$[3,1]$} & {$[3,2]$} & {$[3,3]$} & {$[3,4]$} & {$[3,5]$} & {$[3,6]$} & {$[3,7]$} \\
\hline 1 & 29.31 & 32.03 & 40.18 & 36.97 & 33.78 & 31.97 & 41.09 & 31.51 \\
\hline 3 & 57.53 & 62.71 & 112.80 & 115.20 & 67.11 & 70.30 & 129.57 & 81.66 \\
\hline 4 & 73.50 & 85.99 & 132.49 & 131.92 & 120.95 & 127.74 & 142.85 & 129.57 \\
\hline 6 & 126.91 & 127.47 & 153.62 & 152.45 & 161.54 & 160.16 & 155.07 & 151.92 \\
\hline 7 & 152.43 & 145.18 & 161.75 & 160.62 & 169.26 & 173.87 & 162.59 & 159.27 \\
\hline 8 & 163.39 & 165.20 & 169.78 & 166.56 & 188.88 & 188.48 & 168.15 & 165.15 \\
\hline 11 & 209.46 & 197.61 & 199.41 & 198.05 & 206.67 & 204.39 & 192.98 & 198.97 \\
\hline 12 & 231.38 & 210.38 & 209.47 & 210.81 & 218.58 & 218.08 & 204.42 & 212.14 \\
\hline 13 & 264.22 & 229.08 & 229.06 & 229.97 & 238.15 & 240.89 & 223.55 & 232.26 \\
\hline 14 & 301.63 & 268.32 & 260.55 & 269.19 & 281.07 & 283.37 & 257.35 & 275.56 \\
\hline 15 & 333.58 & 333.58 & 333.58 & 333.58 & 333.58 & 333.58 & 333.58 & 333.58 \\
\hline
\end{tabular}

Table 2. Vectors of characteristic entropy.

\begin{tabular}{|c|c|c|c|c|c|c|c|c|}
\hline Fault Types & $\boldsymbol{H}_{\mathbf{0}}$ & $\boldsymbol{H}_{\mathbf{1}}$ & $\boldsymbol{H}_{\mathbf{2}}$ & $\boldsymbol{H}_{\mathbf{3}}$ & $\boldsymbol{H}_{\mathbf{4}}$ & $\boldsymbol{H}_{\mathbf{5}}$ & $\boldsymbol{H}_{\mathbf{6}}$ & $\boldsymbol{H}_{\mathbf{7}}$ \\
\hline \hline Normal & 1.2501 & 1.1804 & 1.0908 & 1.2253 & 1.2535 & 1.2831 & 1.0701 & 1.1723 \\
\hline Fault I Sig1 & 1.1322 & 0.8013 & 0.6141 & 0.6343 & 0.8991 & 1.0187 & 0.5067 & 0.7057 \\
\hline Fault I Sig2 & 1.1892 & 0.7449 & 0.6050 & 0.5402 & 0.8385 & 0.9015 & 0.5212 & 0.6337 \\
\hline Fault I Sig3 & 1.1890 & 0.7865 & 0.7175 & 0.5687 & 0.9045 & 1.0154 & 0.5424 & 0.6697 \\
\hline Fault II Sig1 & 1.1642 & 0.8966 & 0.8927 & 0.8353 & 1.0801 & 1.2816 & 0.7964 & 0.8895 \\
\hline Fault II Sig2 & 1.1279 & 0.8091 & 0.7306 & 0.7655 & 0.9805 & 1.1783 & 0.6418 & 0.8824 \\
\hline Fault II Sig3 & 1.0885 & 0.6342 & 0.4679 & 0.5494 & 0.7666 & 0.8363 & 0.4075 & 0.6585 \\
\hline
\end{tabular}

Node separation method in Table 1 figured out the section energy of each signal section; then term entropy vectors were calculated according to formula (8) and (9). Table 2 shows the calculation results. According to term entropy vector, elements of normal-signal entropy vector are equally distributed. However, elements of fault-signal entropy vector, usually smaller than that of normal state, are in dispersed distribution. Therefore, the energy distribution of each frequency section under fault state is obviously easier to get disturbed than under normal state. Thus, it can detect circuit breaker faults. Meanwhile, it can be seen that the entropy of fault signals under screw-loosened state is generally smaller than the entropy under time-delayed state.

\subsection{Comparison of Identification Results Between Wave- let Neural Network and BP Neural Network}

On one hand, we made use of tool case to construct wavelet neural network shown in Fig. (1) under the condition of Matlab. Then, we took formula (1) a network equation. Considering the self-adaptive ability of B sample wavelet to data changes, we selected 4th-order B sample wavelet for wavelet function, 8 units for input layers and 3 units for output layers. Data in Table 2 was used as input. After 500 trainings, the error reached expected value of 0.01 . Then, the network training was finished. Table $\mathbf{3}$ shows the output results. On the other hand, we constructed a simple 

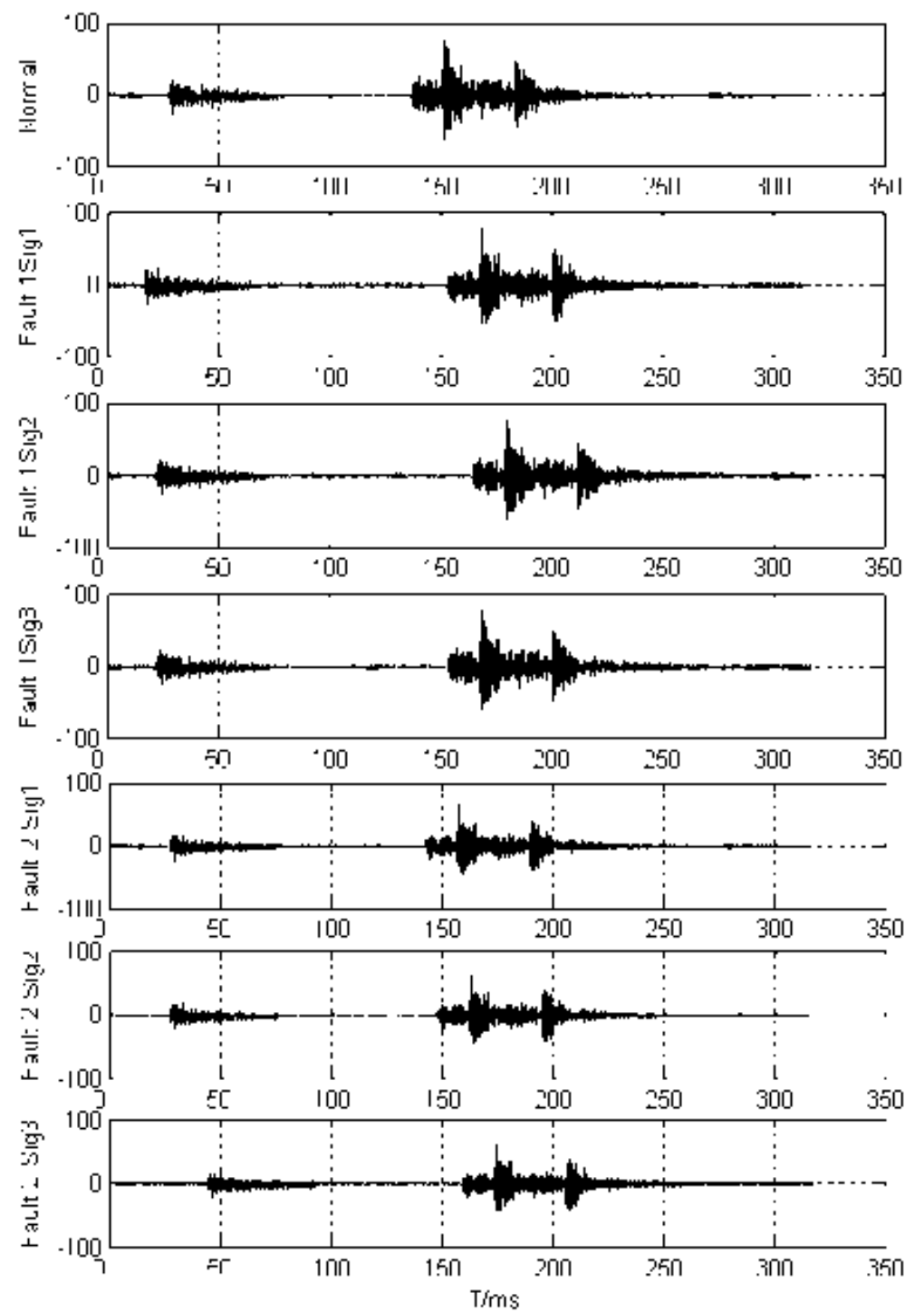

Fig. (2). Original signals of A.

single-hidden-layer BP neural network with 8 neural units for input layers, 3 neural units for output layers. And tansigmoid is the transform function under same Matlab conditions. We chose the number of hidden-layer neural units and step length according to training status. Here we also used data in Fig. (2) to train the network. Given the training function is trained and expected error is 0.01 , after multiple adjustive trainings, the number of hidden-layer neural units is determined to be 10 and step length 0.31 . As a result, there are altogether 4869 steps to finally reach expected error, which nearly takes 4 minutes. Table 4 shows the output results.

Table 3 and Table 4 show the comparison of classification results: wavelet neural network and BP neural network both can achieve accurate identification of fault. Besides, they further prove the effectiveness of feature extraction of this method. However, wavelet neural work has better training speed and classification effect than BP neural network.
Although wavelet neural network and BP neural network have uniform approximation and $L_{2}$ approximation ability, wavelet neural network is a local-approaching network. BP neural network belongs to global-approaching network. Compared with global-approaching network, local-approaching network with fast convergence is easy to adapt to new data, thus avoiding serious extrapolation error. Considering from structure, hidden node function of wavelet neural network is a neural function; weights and hidden thresholds from input layer to hidden layer are corresponding wavelet scaling and translation parameters. As for network structure with $p$ inputs, $h$ hidden nodes and $q$ outputs, the adjustable parameters of wavelet neural network has only $h \times q$ weight coefficients when the range of $j$ and $k$ are determined according to prior knowledge. But BP neural network has $p \times h+h \times q$ input and output weights with $h+q$ thresholds. Therefore, wavelet neural network has fewer adjustable parameters, greatly shortening training time. Considering from the 

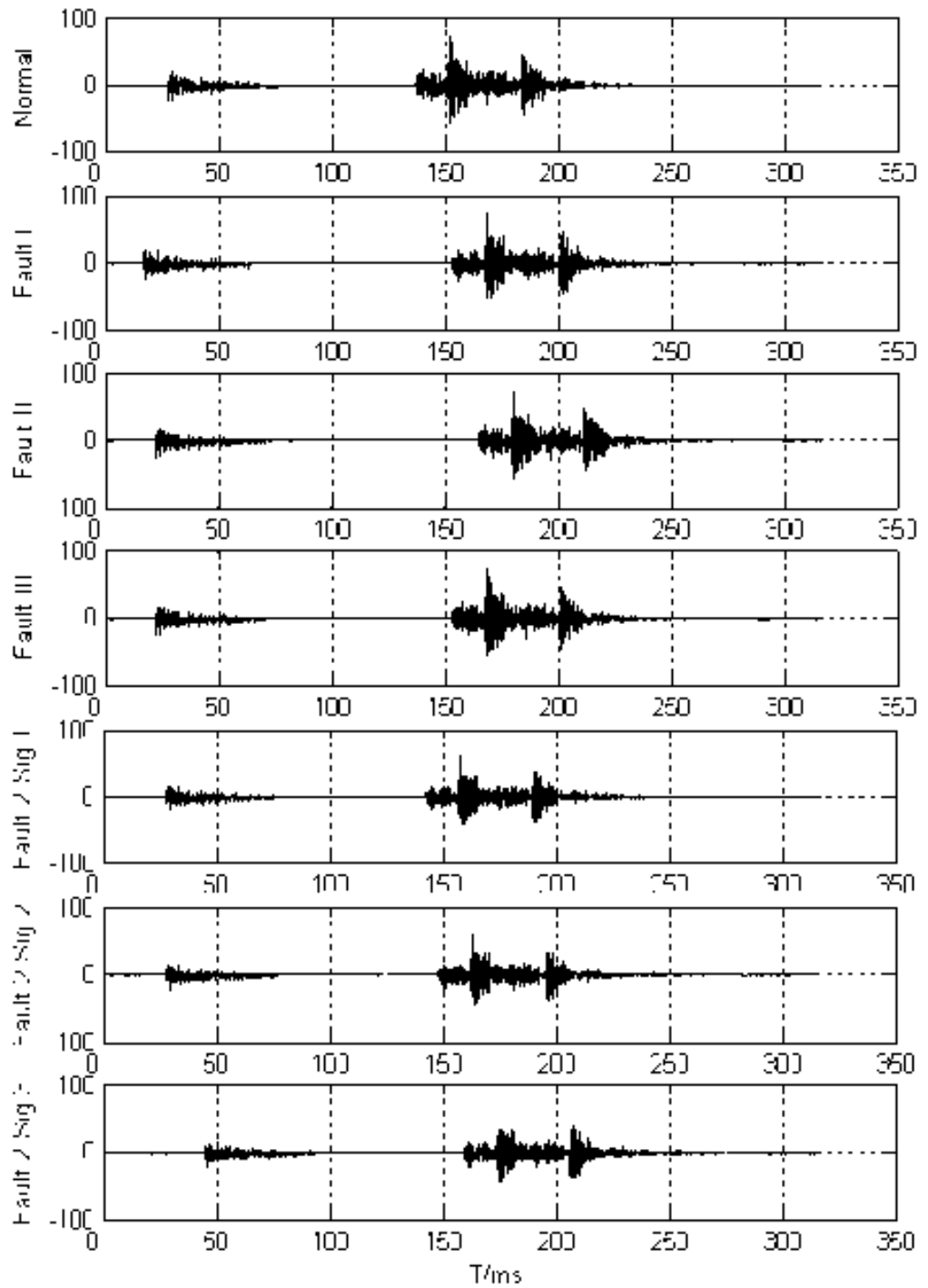

Fig. (3). Signals of A after noise removing.

Table 3. Some testing results of WNN.

\begin{tabular}{|c|c|c|c|c|c|}
\hline Outputs & $\boldsymbol{y}_{\boldsymbol{1}}$ & Expected & $\boldsymbol{y}_{2}$ & Expected & $\boldsymbol{y}_{3}$ \\
\hline \hline Normal & 0.9985 & 1 & -0.0012 & 0 & 0.0017 \\
\hline Normal & 0.9828 & 1 & 0.0025 & 0 & 0.0009 \\
\hline Normal & 0.9917 & 1 & 0.0104 & 0 & -0.0031 \\
\hline Fault I Sig1 & -0.0023 & 0 & 0.9934 & 1 & 0 \\
\hline Fault I Sig2 & -0.0031 & 0 & 0.9887 & 1 & 0 \\
\hline Fault I Sig3 & -0.0026 & 0 & 0.9931 & 1 & 0.0015 \\
\hline
\end{tabular}


Table 3. contd...

\begin{tabular}{|c|c|c|c|c|c|c|}
\hline Outputs & $y_{1}$ & Expected & $y_{2}$ & Expected & $y_{3}$ & Expected \\
\hline Fault I Sig4 & 0.0012 & 0 & 0.9879 & 1 & -0.0014 & 0 \\
\hline Fault I Sig5 & -0.0107 & 0 & 0.9866 & 1 & -0.0083 & 0 \\
\hline Fault I Sig6 & 0.0043 & 0 & 0.9927 & 1 & -0.0019 & 0 \\
\hline Fault I Sig7 & 0.0015 & 0 & 0.9946 & 1 & 0.0042 & 0 \\
\hline Fault I Sig8 & -0.0020 & 0 & 0.9859 & 1 & 0.0110 & 0 \\
\hline Fault II Sig1 & -0.0014 & 0 & -0.0042 & 0 & 0.9982 & 1 \\
\hline Fault II Sig2 & -0.0020 & 0 & -0.0017 & 0 & 0.9951 & 1 \\
\hline Fault II Sig3 & -0.0019 & 0 & -0.0035 & 0 & 0.9941 & 1 \\
\hline Fault II Sig4 & 0.0114 & 0 & -0.0024 & 0 & 0.9768 & 1 \\
\hline Fault II Sig5 & -0.0052 & 0 & 0.0007 & 0 & 0.9849 & 1 \\
\hline Fault II Sig6 & 0.0086 & 0 & 0.0016 & 0 & 0.9855 & 1 \\
\hline Fault II Sig7 & 0.0027 & 0 & -0.0108 & 0 & 0.9914 & 1 \\
\hline Fault II Sig8 & -0.0008 & 0 & -0.0037 & 0 & 0.9776 & 1 \\
\hline
\end{tabular}

Table 4. Some testing results of neural network.

\begin{tabular}{|c|c|c|c|c|c|c|}
\hline Outputs & $y_{1}$ & Expected & $y_{2}$ & Expected & $y_{3}$ & Expected \\
\hline Normal & 0.9472 & 1 & -0.0313 & 0 & 0.0756 & 0 \\
\hline Normal & 0.9711 & 1 & 0.0145 & 0 & 0.0529 & 0 \\
\hline Normal & 0.9526 & 1 & -0.0241 & 0 & 0.0529 & 0 \\
\hline Fault I Sig1 & 0.0733 & 0 & 0.9627 & 1 & -0.0472 & 0 \\
\hline Fault I Sig2 & -0.0461 & 0 & 0.9559 & 1 & -0.0284 & 0 \\
\hline Fault I Sig3 & 0.0197 & 0 & 0.9761 & 1 & 0.0701 & 0 \\
\hline Fault I Sig4 & -0.0436 & 0 & 0.9788 & 1 & -0.0512 & 0 \\
\hline Fault I Sig5 & -0.0318 & 0 & 0.9634 & 1 & -0.0803 & 0 \\
\hline Fault I Sig6 & -0.0098 & 0 & 0.9885 & 1 & 0.0117 & 0 \\
\hline Fault I Sig7 & 0.0181 & 0 & 0.9539 & 1 & -0.0371 & 0 \\
\hline Fault I Sig8 & 0.0602 & 0 & 0.9685 & 1 & -0.0209 & 0 \\
\hline Fault II Sig1 & 0.0721 & 0 & 0.0329 & 0 & 0.9551 & 1 \\
\hline Fault II Sig2 & -0.0387 & 0 & -0.0624 & 0 & 0.9438 & 1 \\
\hline Fault II Sig3 & -0.0196 & 0 & -0.0087 & 0 & 0.9927 & 1 \\
\hline
\end{tabular}


Table 4. contd...

\begin{tabular}{|c|c|c|c|c|c|}
\hline Outputs & $\boldsymbol{y}_{\boldsymbol{1}}$ & Expected & $\boldsymbol{y}_{2}$ & Expected & $\boldsymbol{y}_{3}$ \\
\hline \hline Fault II Sig4 & -0.0228 & 0 & 0.0475 & 0 & 0.9543 \\
\hline Fault II Sig5 & -0.0631 & 0 & -0.0308 & 0 & 0.9596 \\
\hline Fault II Sig6 & 0.0487 & 0 & 0.0523 & 0 & 0.9818 \\
\hline Fault II Sig7 & -0.0288 & 0 & -0.0480 & 0 & 0.9771 \\
\hline Fault II Sig8 & 0.0548 & 0 & 0.0265 & 0 & 0.9628 \\
\hline
\end{tabular}

parameter-learning algorithm, network output and weight coefficient of wavelet neural network are in linear relationship, so weight coefficient can be obtained through linear optimization method. As for multi-resolution orthonormal wavelet networks, there is a unique solution to weight solving equation. Therefore, wavelet neural network achieves good classification effect quickly. Even for signals with noise, it can realize a better identification. Thus, it is very suitable to diagnose HV circuit breaker faults such as environment disturbance.

\section{CONCLUSION}

Based on the introduction of basic principles and training methods of wavelet neural network, the work applied wavelet-packet term entropy method to extract characteristic signals for the training and learning of wavelet neural network. Meanwhile, training and classification effects of waveletpacket term entropy were compared with those of BP neural network method. According to experiments, wavelet neural network is more suitable to identify the status of HV breaker based on vibration signal. Thus, wavelet neural network possess superiority when fast and accurate real-time fault diagnosis is expected.

\section{CONFLICT OF INTEREST}

The author confirms that this article content has no conflict of interest.

\section{ACKNOWLEDGMENT}

This work is supported by natural science foundation of Heilongjiang province of China (E201233).

\section{REFERENCES}

[1] L. Huang, W. Wang, Z. Wu, and L. Xu, "Research on the model of HV SF6 circuit breaker fault diagnosis based on fuzzy theory", In: Proc. Int. Conf. Condition Monitoring and Diagnosis, Beijing, China, pp. 428-431, Apr. 21-24, 2008.

[2] J. J. Ni, C. B. Zhang, and S. Yang, "An adaptive approach based on KPCA and SVM for real-timefault diagnosis of HVCBs", IIEEE Trans. Power Deliv., vol. 26, no. 3, pp. 1960-1971, 2011.
[3] Y. L. Huang, J. J. Wang, W.C. Zhang, M. Al-Dweikat, D. Li, T. Yang, and S. Shao, "A motor-drive-based operating mechanism for high-voltage circuit breaker", IEEE Trans. Power Deliv., vol. 28, no. 4 , pp. 2602-2609, 2013.

[4] M. Runde, G. E. Ottesenet, B. Skyberg, M. Ohlen, "Vibration analysis for diagnostic testing of circuit breakers", IEEE Trans. Power Deliv., vol. 11, no.4, pp. 1816-1823, 1996.

[5] X. G. Hu, J. M. Dai, Y.C. Yan, "The fault diagnosis of high voltage breakers based on wavelet singularity detection", Proc CSEE, vol. 21 , no. 5, pp. 67-70, 2001.

[6] D. S. S. Lee, B. J. Lithgow, R. E. Morrison, "New fault diagnosis of circuit breakers", IEEE Trans. Power Deliv., vol. 18, no. 2, pp. 454-459, 2003.

[7] K.N. Reddy, and V. Ravi, "Differential evolution trained kernel principal component WNN and kernel binary quantile regression: Application to banking", Knowl.-Based Syst., vol. 39, pp. 45-56, 2013.

[8] S. Poungponsri, X. H. Yu, "An adaptive filtering approach for electrocardiogram (ECG) signal noise reduction using neural networks", Neurocomputation, vol. 117, no. 6, pp. 206-213, Oct. 2013.

[9] Z. Zainuddin, and P. Ong, "Reliable multiclass cancer classification of microarray gene expression profiles using an improved wavelet neural network" Expert Syst. Appl., vol. 38, no. 11, pp. 1371113722, Oct. 2011.

[10] H. K. Sahoo, P. K. Dash, and N. P. Rath, "NARX model based nonlinear dynamic system identification using low complexity neural networks and robust $\mathrm{H} \infty$ filter" Appl. Soft Comput., vol.13, no.7, pp. 3324-3334, 2013.

[11] R. H. Abiyev, O. Kaynak, E. Kayacan, "A type-2 fuzzy wavelet neural network for system identification and control", J. Franklin Inst., vol. 350, no. 7, pp. 1658-1685, Sept.2013.

[12] J. D. Zheng, J. S. Cheng, Y. Yang, and S. Luo, "A rolling bearing fault diagnosis method based on multi-scale fuzzy entropy and variable predictive model-based class discrimination", Mech. Mach. Theory, vol. 78, pp. 187-200, August 2014.

[13] A. B. Ming, Z. Y. Qin, W. Zhang, and F. L. Chu, "Spectrum autocorrelation analysis and its application to fault diagnosis of rolling element bearings", Mech. Syst. Signal Proces., vol. 41, no.1-2, pp. 141-154, Dec. 2013.

[14] Spagnoli, L. Montanari, B. Basu, "Nonlinear damage identification in fiber-reinforced cracked composite beams through time-space wavelet analysis", Proc Mater. Sci., vol. 3, pp. 1579-1584, 2014.

[15] H. R. F. Masoumi, M. Basri, A. Kassim, D. K. Abdullaha, Y. Abdollahib, S. S. Abd Gania, M. Rezaeec, "Optimization of process parameters for lipase-catalyzed synthesis of esteramines-based esterquats using wavelet neural network (WNN) in 2-liter bioreactor", J. Indust. Eng. Chem., vol. 20, no. 4, pp. 1973-1976, 25 July 2014.

[16] L. J. Sun, M. L. Liu, J. J. Zhen and G. Z. Ye. "A new fault diagnosis method for hv circuit breakers based on wavelet packet-neural network", In: $20116^{\text {th }}$ IEEE Conference on Industrial Electronics and Applications, pp. 838-844, 2011. 
[17] L. J. Sun, M.L. Liu, H.B. Qian and J.J. Zhen, "A new method to fault diagnosis for circuit breakers based on characteristic entropy of wavelet packet", 2010 International Conference on Intelligent System Design and Engineering Application, pp. 838-841, 2010.
[18] L. J. Sun, M. L. Liu, and J. J. Zhen, "Characteristic entropy tree of wavelet packet and its application in fault diagnosis", J. Harbin Ins. Technol. (New Series), vol.18, pp. 293-298, 2011.

Received: December 15, 2014

Revised: January 04, 2015

Accepted: February 25, 2015

(C) Mingliang et al.; Licensee Bentham Open.

This is an open access article licensed under the terms of the Creative Commons Attribution Non-Commercial License (http://creativecommons.org/licenses/by$\mathrm{nc} / 3.0 /$ ) which permits unrestricted, non-commercial use, distribution and reproduction in any medium, provided the work is properly cited. 